\title{
ASPECTOS PARTICULARES DEL DISEÑO DE MUROS
}

\author{
(SPECIAL CHARACTERISTICS OF WALL DESIGN)
}

J. Calavera Ruiz, Prof. Dr. Ingeniero de Caminos

J. Ley Urzaiz, Ingeniero de Caminos

Cátedra de Edificación y Prefabricación de la E.T.S. de Ingenieros de Caminos de Madrid/España

Fecha de recepción: $12-X 11-88$

\section{RESUMEN}

A partir de la Instrucción EH-82, en el cálculo de los distintos elementos estructurales, lo que se ponderan por el coeficiente de seguridad $\gamma_{t}$ no son los esfuerzos en dichos elementos, sino las acciones que dan lugar a ellos. Esta importante diferencia conceptual, en la mayoria de las estructuras que manejamos, no conduce a diferencias numéricas grandes entre los resultados obtenidos por uno u otro método. En el presete trabajo se muestra cómo en el caso de los muros, las diferencias son grandes y la variación del nivel de seguridad importante si se ponderan los esfuerzos y no las acciones.
SUMMARY

This paper deals about the difference between designing some parts of the retaining walls, multiplicand the actual loads and active confinement pressures by the appropiate load factors (the correct and mandatory method) and the obtaining the bearing pressures, and the design of those parts working with the bearing pressures obtained multiplicand the bearing pressures under service load conditions, by the load factor. The study shows the important difference between the results obtained by means of the two methods.

\section{INTRODUCCION}

Al implantar en España el cálculo semiprobabilista de las estructuras de hormigón mediante el método de los estados límites, se adoptaron tres coeficientes parciales de seguridad:

- El $\gamma_{c}$ que divide a la resistencia característica del hormigón.

- El $\gamma_{\mathrm{s}}$ que divide al límite elástico característico del acero.

- El $\gamma_{f}$ que pondera los esfuerzos actuantes; multiplicándolos por valores mayores que la unidad cuando su efecto es desfavorable y por valores menores que la unidad, incluso nulos, cuando su efecto es favorable.
Sin embargo, en relación con este tercer coeficiente, el $\gamma_{f}$, las Instrucciones sucesivas adoptan dos posiciones diferentes según la época:

- Hasta la Instrucción EH-73, inclusive, a partir de los valores característicos de las acciones que designaremos simbólicamente por $F_{k}$, se calculan los esfuerzos:

$$
E_{k}=\varphi\left(F_{k}\right)
$$

- y de ellos se obtenían los esfuerzos de cálculo:

$$
E_{d}=\gamma_{f} \times E_{k}=\gamma_{f} \times \varphi\left(F_{k}\right)
$$

- A partir de la Instrucción EH-82 se adopta un sistema diferente que se mantiene en EH-88. 
Lo que se ponderan son las acciones y no los esfuerzos, porque realmente son las acciones las que son variables aleatorias. Ello conduce a que:

$$
\begin{gathered}
F_{d}=\gamma_{f} \times F_{k} \\
E_{d}=\varphi\left(\gamma_{f} \times F_{k}\right)=\varphi\left(F_{d}\right)
\end{gathered}
$$

En la inmensa mayoria de las estructuras que manejamos

$$
\gamma_{\mathrm{f}} \times \varphi\left(\mathrm{F}_{\mathrm{k}}\right)=\varphi\left(\gamma_{\mathrm{f}} \times \mathrm{F}_{\mathrm{k}}\right)
$$

y en ellas, en la práctica, ambos sistemas conducen a los mismos resultados, aunque la importante diferencia conceptual entre ambos sistemas permanezca.

Sin embargo, en algunas estructuras la diferencia entre ambos sistemas no sólo es conceptual sino también numérica. Un caso importante es el de los muros de contención. En ellos, la práctica habitual (figura 1), es obtener los valores de los esfuerzos bajo la acción del empuje característico (o de servicio) $E_{k}$, que provoca las tensiones características (o de servicio) $\sigma_{\mathrm{k}} \mathrm{y}$ posteriormente obtener los esfuerzos de cálculo multiplicando los debidos a $E_{k}$ por $\gamma_{f}$

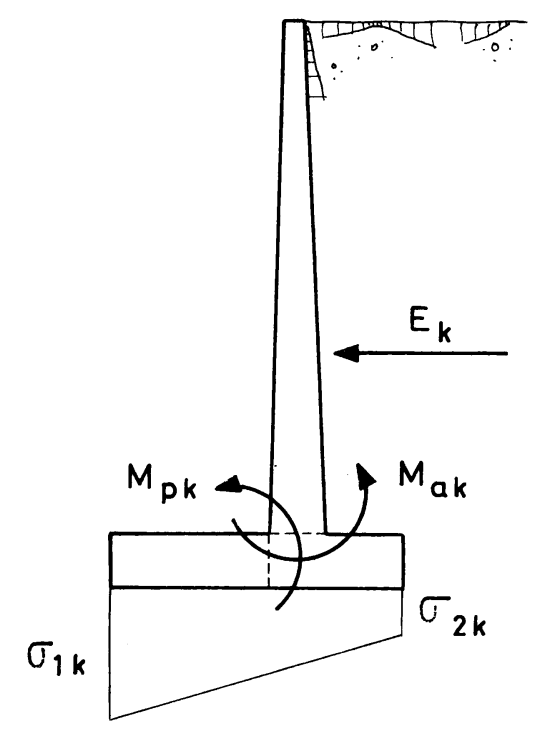

Fig. 1

$$
\begin{gathered}
M_{a k}=\varphi\left(E_{k}\right) \\
M_{a d}=\gamma_{f} \times M_{a k}=\gamma_{f} \times \gamma_{1}\left(E_{k}\right) \\
M_{p k}=\varphi\left(\sigma_{k}\right) \\
M_{p d}=\gamma_{f} \times M_{p k}=\gamma_{f} \times \varphi_{2}\left(\sigma_{k}\right)
\end{gathered}
$$

Sin embargo, y de acuerdo con las Instrucciones EH-82 y EH-88, lo correcto es calcular el empuje mayorado $E_{d}$ $y$ las tensiones $\sigma_{\mathrm{d}}$ que éste provoca, tal como se indica en la figura 2

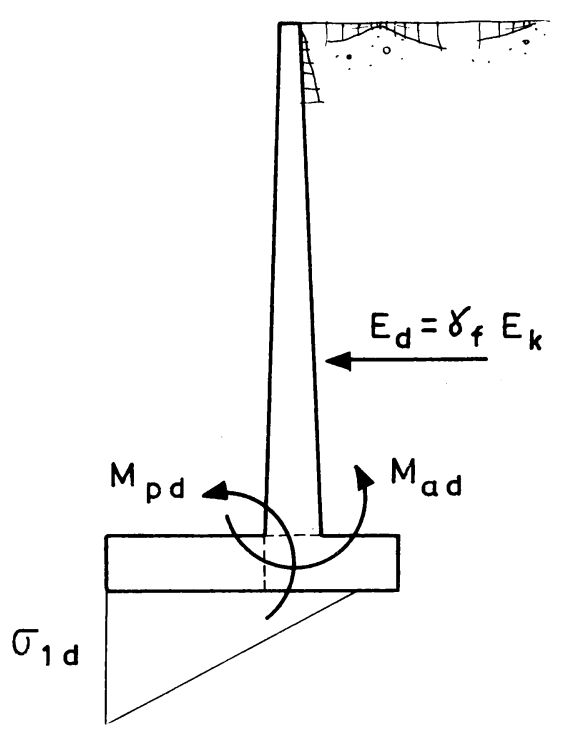

Fig. 2

$$
\begin{gathered}
M_{a d}=\varphi_{1}\left(E_{d}\right)=\gamma_{f} \varphi_{1}\left(E_{k}\right) \\
M_{p d}=\varphi_{n}\left(\sigma_{d}\right) \neq \gamma_{f} \times \varphi_{2}\left(\sigma_{k}\right)
\end{gathered}
$$

Es inmediato observar que el valor de $M_{\mathrm{ad}}$ del momento en el arranque del alzado en el caso de la figura 2 coincide exactamente con el obtenido en el caso de la figura 1, pero no ocurre lo mismo con el momento en la puntera $M_{p}$, ya que no hay proporcionalidad entre el empuje $E$ y, las tensiones $\sigma$, e incluso puede ocurrir, según los casos, lo indicado en la figura 2, es decir que el diagrama de tensiones $\sigma_{\mathrm{d}}$ sea ahora triangular en lugar de trapecial, con lo que la función $\varphi_{2}$ ni siquiera será válida, sino que tendremos una nueva función $\varphi$.

En lo que sigue, y a la vista de lo expuesto analizamos la diferencia entre los momentos de cálculo de la puntera con uno y otro método de cálculo.

Seguimos un método general de cálculo de muros expuesto en la referencia (3) con la notación allí indicada.

\section{HIPOTESIS DE PARTIDA}

Sea un muro de puntera y talón tipo como el de la figura 3. 


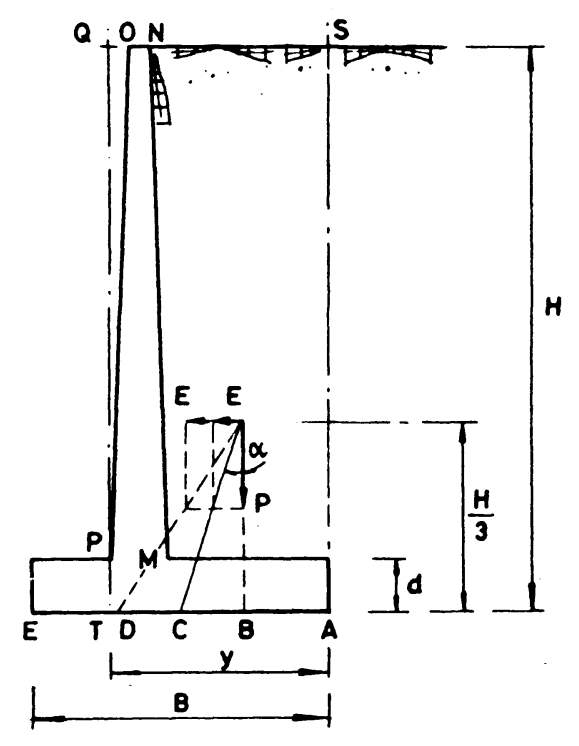

Fig. 3

Con objeto de reducir el número de variables en el problema a estudiar, introduciremos las siguientes simplificaciones:

a) Despreciar el peso de la puntera, que es escaso en los muros normales.

b) Sustituir el peso del alzado MNOP de Hormigón (con densidad en la realidad inferior a $2,5 \mathrm{t} / \mathrm{m}^{3}$ indicada en la Instrucción EH 88) por el rectángulo MNQP, considerado como ocupado por el suelo a contener. La hipótesis es suficientemente aproximada, tanto en el valor del peso como en la posición del centro de gravedad.

c) Suponer un muro virtual TQ, de trasdós vertical y sometido al empuje producido por el relleno, para el que se considera una densidad aparante $r_{h}=1,8$ $\mathrm{t} / \mathrm{m}^{3}$.

d) Suponer un peso vertical total $P$, correspondiente al macizo de suelo ATQS, con un peso específico $r$ ' intermedio entre el del suelo y el hormigón. El valor de $r^{\prime}$ es función de la relación $\mathrm{d} / \mathrm{H}$, siendo $\mathrm{r}^{\prime}=$ $1,8+(2,4-1,8) \mathrm{d} / \mathrm{H}$ (unidades en $\mathrm{m}$ y $\mathrm{t}$ ). Como generalmente el valor de $\mathrm{d} / \mathrm{H}$ difiere poco en la práctica de 0,1 , adoptaremos para r' el valor 1,86.

e) Para el cálculo del empuje emplearemos la fórmula de RANKINE:

$$
E_{k}=\frac{1}{2} \times r_{h} \times H^{2} \times \frac{1-\operatorname{sen} \varnothing}{1+\operatorname{sen} \varnothing}=K \times H^{2}
$$

suponiendo el trasdós drenado y siendo $r_{h}$ la densidad del suelo.
Dada la finalidad que perseguimos estudiaremos el uso de dos hipótesis, una primera suponiendo bajo la zapata del muro unas tensiones en servicio, y hallando entonces un momento que posteriormente se mayorará para obtener el momento de cálculo, y una segunda (la que debía realizarse en una buena práctica) suponiendo un reparto de tensiones de cálculo obtenido al mayorar el empuje, y calculando entonces el momento de cálculo debido a esas presiones.

\section{DESARROLLO DE LOS CALCULOS}

En general y de acuerdo con la figura 3 se tiene:

$$
\begin{gathered}
E_{k}=K \times H^{2} \\
P=1,86 \times H \times y \\
\frac{E}{P} \operatorname{tg} \alpha=v \\
B C=v \times H / 3 \\
A C=v \times H / 3+y / 2
\end{gathered}
$$

Sustituyendo en [15] los valores de $E_{k}$ y $P$ dados en [13] $y$ [14] se obtiene $v=\frac{K \times H}{1,86 \times y}$

Llamando $e_{k}$ a la excentricidad de la resultante (obtenida sin mayorar el empuje $E_{k}$, respecto del centro del cimiento (positiva hacia la puntera) se obtiene:

$$
e_{k}=A C-B / 2=\frac{K \times H^{2}}{5,58 \times y}+y / 2-B / 2
$$

expresando esta excentricidad en forma adimensional:

$e_{k} / H=\frac{K}{5,58 \times y / H-B / H}+0,5 \times(y / H / B / H)-0,5$

Los valores de $e_{k} / B$ oscilan desde 0 (resultante centrada y por tanto reparto uniforme de presiones sobre el suelo) y $e_{k} / B=0,333$ que suele considerarse un límite superior práctico.

Si consideramos mayorado el empuje desde el valor de servicio hasta $E_{d}=1,6 \times E_{k}$ (hay que observar que el valor 1,6 no es un valor fijo, se toma el valor 1,6 que es el más usual para establecer una comparación entre los dos métodos que estudiamos).

Llamando $e_{d}$ a la excentricidad de la resultante calculada con $E_{d}$ tenemos:

$$
e_{d}=A D-B / 2
$$


$A D=B D+y / 2=1,6 \times H / 3 \times v+y / 2$

$A D=\frac{K \times H^{2}}{3,49 \times y}+y / 2$

$e_{d}=\frac{K \times H^{2}}{3,49 \times y}+y / 2-B / 2$

expresándolo en forma adimensional:

$$
\begin{aligned}
e_{d} / B= & \frac{K}{3,49 \times y / H \times B / H}+0,5 \times \\
& \times(y / H / B / H)-0,5
\end{aligned}
$$

Una vez que hemos obtenido las expresiones de las excentricidades obtendremos las distribuciones de presiones bajo las zapatas de los muros con forma triangular o trapecial según el valor de las excentricidades como veremos a continuación:

\section{1. ${ }^{\text {er Caso: }}$}

$$
e_{k} / B>=1 / 6
$$

La distribución de tensiones bajo la zapata es triangular bajo el empuje sin mayorar y mayorado (es decir en este caso $e_{d} / B>=1 / 6$ ).

Calculemos el momento actuante en la sección PT (ver figura 3) en el supuesto de empuje sin mayorar:

De la figura $\sigma_{\mathrm{k}}=\frac{2 \times \mathrm{P}}{3 \times \mathrm{EC}} ; \mathrm{EC}=\mathrm{B}-\mathrm{AC}=\mathrm{B}-$

$$
\begin{gathered}
-\frac{\mathrm{K} \times \mathrm{H}^{2}}{5,58 \times \mathrm{y}}-\mathrm{y} / 2 \\
\sigma_{\mathrm{k}}=\frac{2 \times 1,86 \times \mathrm{H} \times \mathrm{y}}{3 \times\left(\mathrm{B}-\left(\left(\mathrm{K} \times \mathrm{H}^{2}\right) /(5,58 \times \mathrm{y})\right)-\mathrm{y} / 2\right.}
\end{gathered}
$$

Se pueden presentar dos situaciones:

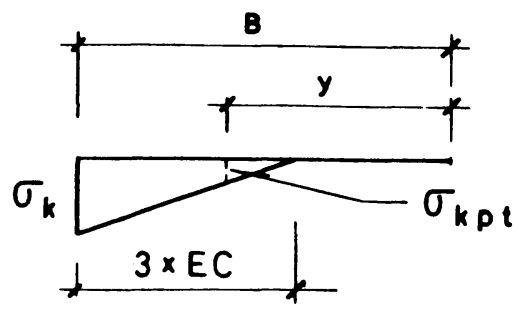

1.A.1: $3 \times E C+y>B$

$\sigma_{k} / H=\frac{1,24}{(B / H / y / H)-\left(K /\left(5,58 \times(y / H)^{2}\right)-0,5\right.}$

$3 \times E C / H=3 \times\left(B / H-\frac{K}{5,58 \times y / H}-\frac{y / H}{2}\right)$

$\sigma_{\mathrm{k}} \mathrm{PT} / \mathrm{H}=\frac{\sigma_{\mathrm{k}} / \mathrm{H}}{3 \times \mathrm{EC} / \mathrm{H}} \times\left(\frac{3 \mathrm{EC}}{\mathrm{H}}-\mathrm{B} / \mathrm{H}+\mathrm{y} / \mathrm{H}\right)$

El valor que nos interesa $M_{p k}$, será igual a:

$M_{\mathrm{pk}}=1 / 6 \times\left(2 \times \sigma_{\mathrm{k}}+\sigma_{\mathrm{k}} \mathrm{PT}\right) \times(\mathrm{B}-\mathrm{y})^{2}$

El valor que tradicionalmente (e incorrectamente) se utiliza para diseñar la sección PT del muro es:

$M_{p d}=1,6 \times M_{p k}$

1.A.2: $3 \times E C+y<=B$

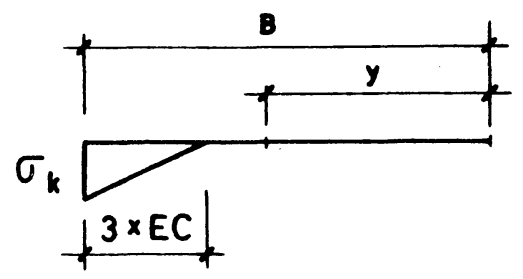

$\sigma_{\mathrm{k}} / \mathrm{H}=\frac{\cdot 1,24}{(\mathrm{~B} / \mathrm{H} / \mathrm{y} / \dot{\mathrm{H}})-\left(\mathrm{K} /\left(5,58 \times(\mathrm{y} / \mathrm{H})^{2}\right)-0,5\right.}$

$3 \times E C / H=3 \times\left(B / H-\frac{K}{5,58 \times y / H}-\frac{y / H}{2}\right)$

$M_{p k}=\frac{1}{2} \times \sigma_{k} \times 3 \times E C \times\left(B \times y-\frac{3 \times E C}{3}\right)$

El valor que tradicionalmente (e incorrectamente) se utiliza para diseñar en este caso la sección PT del muro es:

$M_{p d}=1,6 \times M_{p k}$

Veamos ahora el valor que obtendríamos de considerar la distribución de presiones resultante de mayorar el empuje (que seria lo correcto).

Como ya se ha dicho al ser $e_{k} / B>1 / 6 ; e_{d} / B$ es también mayor que $1 / 6$ con lo que la distribución de tensiones es triangular. 
Tendremos también dos casos posibles:

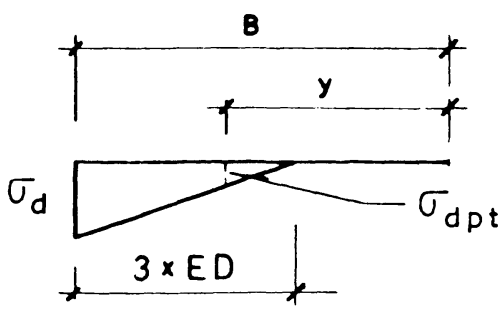

1.B.1: $3 \times E D+y>B$

De la figura $\sigma_{d}=\frac{2 \times P}{3 \times E D} ; E D=B-A D=B-$

$$
-\frac{\mathrm{K} \times \mathrm{H}^{2}}{3,49 \times \mathrm{y}}-\mathrm{y} / 2
$$

$\sigma_{\mathrm{d}} / \mathrm{H}=\frac{1,24}{(\mathrm{~B} / \mathrm{H} / \mathrm{y} / \mathrm{H})-\left(\mathrm{K} /\left(3,49 \times(\mathrm{y} / \mathrm{H})^{2}\right)-0,5\right.}$

$3 \times E D / H=3 \times\left(B / H-\frac{K}{3,49 \times y / H}-\frac{y / H}{2}\right)$

$\sigma_{\mathrm{d}} \mathrm{PT} / \mathrm{H}=\frac{\sigma_{\mathrm{d}} / \mathrm{H}}{3 \times E C / H} \times\left(\frac{3 E D}{H}-\mathrm{B} / \mathrm{H}+\mathrm{y} / \mathrm{H}\right)$

El valor que nos interesa $M_{\mathrm{Pd}}$, será igual a:

$$
\mathrm{M}_{\mathrm{PD}}=1 / 6 \times\left(2 \times \sigma_{\mathrm{d}}+\sigma_{\mathrm{d}} \mathrm{PT}\right) \times(\mathrm{B}-\mathrm{y})^{2}
$$

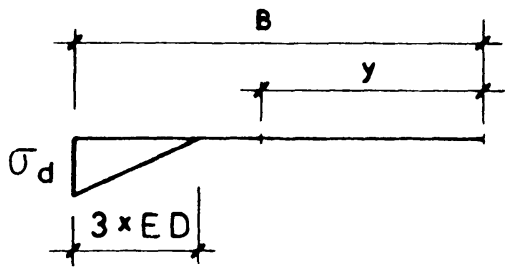

1.B.2: $3 \times E D+y \leq B$

$$
\sigma_{\mathrm{d}} / \mathrm{H}=\frac{1,24}{(\mathrm{~B} / \mathrm{H} / \mathrm{y} / \mathrm{H})-\left(\mathrm{K} /\left(3,49 \times(\mathrm{y} / \mathrm{H})^{2}\right)-0,5\right.}
$$

$3 \times E D / H=3 \times\left(B / H-\frac{K}{3,49 \times y / H}-\frac{y / H}{2}\right)$

$M_{P d}=\frac{1}{2} \times \sigma_{d} \times 3 \times E D \times\left(B \times y-\frac{3 \times E D}{3}\right)$

Valor con el que en este caso diseñariamos la sección PT del muro.

\section{2. ${ }^{\circ}$ Caso}

Es el que se produce si e/B $<=1 / 6$ (en este caso $e_{d} / B$ puede ser $>=0<<1 / 6$ ), la distribución de tensiones bajo la zapata es trapecial en el caso de calcular el momento debido a las presiones en servicio.

El momento se calcula mediante el siguiente proceso:

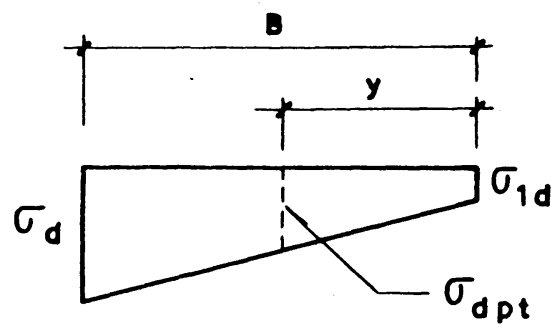

$\sigma_{k} / H=1,86 \times \frac{y / H}{B / H} \times(1+6 \times e / B)$

$\sigma_{\mathrm{k} 1} / \mathrm{H}=1,86 \times 2 \times \frac{\mathrm{y} / \mathrm{H}}{\mathrm{B} / \mathrm{H}} \times \sigma_{\mathrm{k}} / \mathrm{H}$

$\sigma_{\mathrm{kPT}} / \mathrm{H}=\sigma_{\mathrm{k} 1} / \mathrm{H}+\frac{\left(\sigma_{\mathrm{k}} / \mathrm{H}-\sigma_{\mathrm{k} 1} / \mathrm{H}\right)}{\mathrm{B} / \mathrm{H}}$

El momento en la sección PT será entonces:

$M_{p k}=1 / 6 \times\left(2 \times \sigma_{k}+\sigma_{k P T}\right) \times(B-y)^{2}$

y el momento que en este caso tradicionalmente se utiliza para calcular la armadura en la sección PT es: $M_{P d}=1,6 \times M_{p k}$

Si hacemos el cálculo del momento debido a las presiones obtenidas de mayorar el empuje, tendremos distribución de tensiones triangular o trapecial en función de que el valor de la excentricidad $e_{d} / B$ sea mayor 0 menor $1 / 6$. Si $e_{d} / B$ es mayor que $1 / 6$ estamos en el caso 1.B ya estudiado, si es menor la distribución de tensiones es trapecial y el cálculo del momento es como sigue:

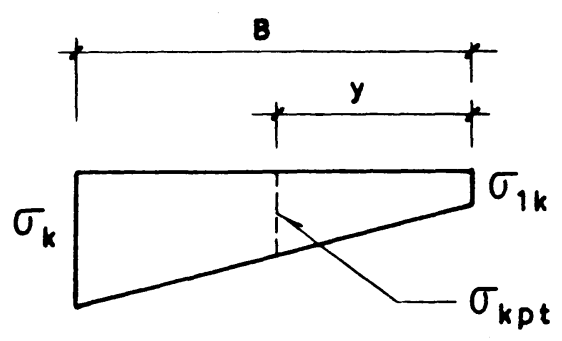

$\sigma_{\mathrm{d}} / \mathrm{H}=1,86 \times \frac{\mathrm{y} / \mathrm{H}}{\mathrm{B} / \mathrm{H}} \times\left(1+6 \times \mathrm{e}_{\mathrm{d}} / \mathrm{B}\right)$ 
$\sigma_{\mathrm{d} 1} / \mathrm{H}=1,86 \times 2 \times \frac{\mathrm{y} / \mathrm{H}}{\mathrm{B} / \mathrm{H}} \times \sigma_{\mathrm{d}} / \mathrm{H}$

$\sigma_{\mathrm{dPT}} / \mathrm{H}=\sigma_{\mathrm{d} 1} / \mathrm{H}+\frac{\left(\sigma_{\mathrm{d}} / \mathrm{H}-\sigma_{\mathrm{d} 1} / \mathrm{H}\right)}{\mathrm{B} / \mathrm{H}}$

El momento en la sección PT será entonces:

$M_{P d}=1 / 6 \times\left(2 \times \sigma_{d}+\sigma_{d P T}\right) \times(B-y)^{2}$

\section{RESUMEN DE RESULTADOS}

Todo lo anterior se resume en la figura 4, en un ábaco en función de los parámetros adimensionales y/H, $\mathrm{B} / \mathrm{H}$.

Las curvas expresan la relación de momentos flectores en puntera calculados por el procedimiento correcto (el prescrito en EH-82 y EH-88) y el habitual. Los valores de $\mathrm{R}$ mayores que 1 , corresponden a descensos de seguridad, respecto a lo indicado en la Instrucción.
Es fácil comprobar que existen en la práctica muros con valores $\mathrm{B} / \mathrm{H}=0,45$ y valores de $\mathrm{y} / \mathrm{H}$ alrededor de $0,30-0,40$, en los que $R$ es del orden de 1,75 . Esto equivale a aceptar que en pura teoría semiprobabilista estamos proyectando muros que con el nuevo criterio tienen valores de $\gamma_{f}$ de 0,9 a 1,1 en lugar de 1,5 ó 1,6. La ausencia de experiencia patológica de fallos de muros por rotura de puntera hace conveniente reflexionar sobre el valor de $\gamma_{f}$ en muros de contención.

\section{BIBLIOGRAFIA}

(1) "Instrucción EH-88 para el proyecto y la ejecución de obras de Hormigón armado o en masa”. M.O.P.U., Madrid 1988.

(2) CALAVERA, J.; CABRERA, A.: "Un método para el proyecto de muros de contención". Informes de la Construcción, n. 210 , mayo 1969.

(3) CALAVERA, J.: "Muros de Contención y Muros de Sótano". INTEMAC, 1987 Madrid.

\section{CURVAS DE INSEGURIDAD R $=\frac{M_{p d}\left(\gamma_{f} \cdot E_{k}\right)}{\gamma_{f} \cdot M_{p k}\left(E_{k}\right)}$}

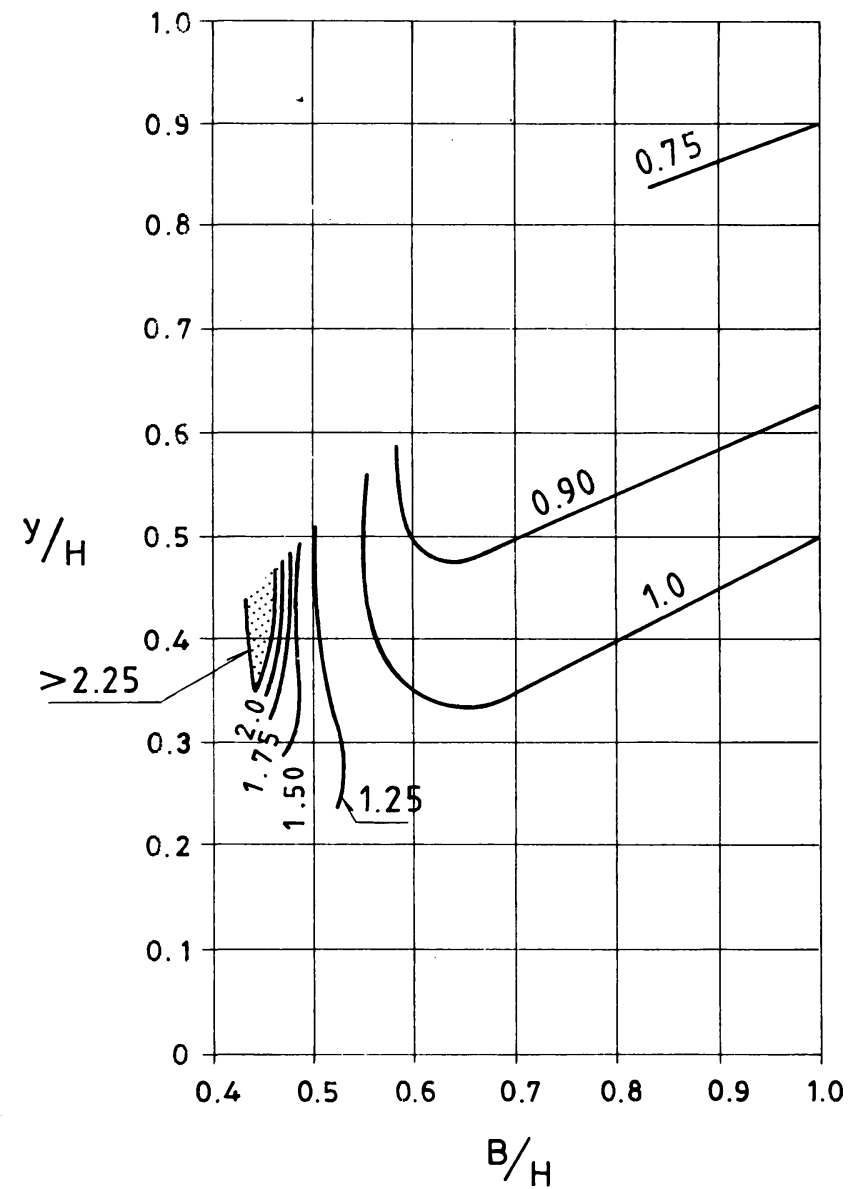

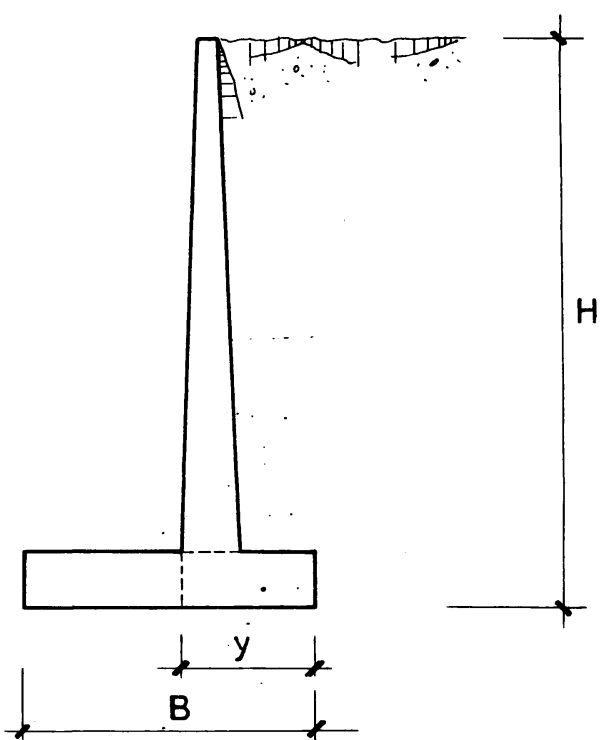

Fig. 4 